\title{
BMJ Open Supporting survivors of sexual violence: protocol for a mixed-methods, co- research study of the role, funding and commissioning of specialist services provided by the voluntary sector in England
}

Gill Combes (1) , ${ }^{1}$ Sarah Damery, ${ }^{1}$ Clare Gunby, ${ }^{1}$ Jenny Harlock, ${ }^{2}$ Louise Isham, ${ }^{1}$ Alice Jones, ${ }^{3}$ Fay Maxted, ${ }^{4}$ Priti Parmar, ${ }^{1}$ Jason Schaub, ${ }^{5}$ Deb Smith, ${ }^{1}$ Julie Taylor, ${ }^{6,7}$ Caroline Bradbury-Jones ${ }^{6}$

To cite: Combes G, Damery S, Gunby $\mathrm{C}$, et al. Supporting survivors of sexual violence: protocol for a mixed-methods, co-research study of the role, funding and commissioning of specialist services provided by the voluntary sector in England. BMJ Open 2019;9:e035739. doi:10.1136/ bmjopen-2019-035739

- Prepublication history for this paper is available online. To view these files, please visit the journal online (http://dx.doi org/10.1136/bmjopen-2019035739).

Received 14 November 2019 Revised 25 November 2019 Accepted 26 November 2019

Check for updates

(C) Author(s) (or their employer(s)) 2019. Re-use permitted under CC BY-NC. No commercial re-use. See rights and permissions. Published by BMJ.

For numbered affiliations see end of article.

Correspondence to

Dr Louise Isham;

I.j.isham@bham.ac.uk

\section{ABSTRACT}

Introduction The voluntary sector provides a range of specialist services to survivors of sexual violence, many of which have evolved from grass roots organisations responding to unmet local needs. However, the evidence base is poor in terms of what services are provided to which groups of survivors, how voluntary sector specialist (VSS) services are organised and delivered and how they are commissioned. This will be the first national study on the role of the voluntary sector in supporting survivors in England.

Methods and analysis This study uses an explanatory sequential naturalistic mixed-methods design with two stages. For stage 1, two national surveys of providers' and commissioners' views on designing and delivering VSS services will facilitate detailed mapping of service provision and commissioning in order to create a taxonomy of VSS services. Variations in the national picture will then be explored in stage 2 through four in-depth, qualitative case studies using the critical incident technique to explain the observed variations and understand the key contextual factors which influence service provision. Drawing on theory about the distinctive service contribution of the voluntary sector, survivors will be involved as coresearchers and will play a central role in data collection and interpretation.

Ethics and dissemination Ethical approval has been granted by the University of Birmingham research ethics committee for stage 1 of the project. In line with the sequential and co-produced study design, further applications for ethical review will be made in due course. Dissemination activities will include case study and end-of-project workshops; good practice guides; a policy briefing; project report; bitesize findings; webinars; academic articles and conference presentations. The project will generate evidence about what survivors want from and value about services and new understanding about how VSS services should be commissioned and provided to support survivors to thrive in the long term.
Strengths and limitations of this study

- This will be the first national study on the role of the voluntary sector in supporting survivors, addressing an important gap in current evidence about what survivors want and value from voluntary sector specialist (VSS) services, how VSS and public sector services link together and how VSS services are commissioned.

- The study's explanatory, sequential naturalistic mixed-methods design will generate robust, nuanced data about VSS services for survivors. The findings will support local agencies to deliver national policy on providing lifelong support to survivors of sexual violence.

- Survivors' views and needs foreground this study. Survivors play a critical role in the design, development and delivery of the study as co-researchers, advisors and co-applicants.

- Analysis of the four case study sites will produce rich but context-specific, non-generalisable data about the provision and commissioning of VSSservices for survivors.

\section{BACKGROUND}

Sexual violence is one of the most pervasive forms of violence that deprives women, men and children of their dignity and violates their human rights. ${ }^{1}$ Sexual violence most often occurs in the context of familial and intimate partner relationships and co-occurs with other forms of abusive, controlling and violent behaviour. ${ }^{2}$ In 2017/2018, 150732 sexual offences were reported to police in England, a 24\% increase on the previous year and the highest figure since records began, ${ }^{3}$ despite most sexual offences still not 
being reported to official authorities. Just over one-third of those offences were for rape. ${ }^{3}$ These numbers do not include sexual violence perpetrated against children and older people.

Survivors of sexual violence can and do recover, however, there can be multiple, long-term effects on health, well-being and social life. In England, the cost of sexual violence is estimated to be over $£ 8$ billion a year and the cost of each rape is $£ 96000 .^{4}$ Rates of posttraumatic stress disorder are highest for sexual violence compared with all other traumatic events. ${ }^{5}$ Depression, anxiety, suicide, self-harm, alcohol/drug abuse and sexually transmitted infection rates are all high ${ }^{67}$ and impacts on mortality have been found. ${ }^{8}$ Survivors report adverse effects on relationships with partners, family and friends and on their confidence/ability to work ${ }^{9}$ and participate in social/community life. Given current and projected rises in sexual violence ${ }^{10}$ and the cost to survivors and society if needs go unmet, there is a critical need for high-quality research about the nature and availability of specialist support services for survivors. This protocol outlines a study that will address this need.

\section{National policy and commissioning landscape}

Historically, the voluntary sector has played a key role in providing specialist services to survivors of sexual violence. In the last decade, there has been increasing national recognition that voluntary sector sepcialist (VSS) services are essential in providing crisis and longer-term support, enabling survivors to recover their confidence and thrive in the longer term. ${ }^{41-14}$ Although services vary from area to area, they often include: crisis and longer-term counselling/therapy; telephone helplines; face-to-face advice; ; play therapy for children; practical support in accessing other services; and, support groups and social activities.

As a result of changes to the structure and funding of health ${ }^{15}$ and criminal justice systems in England, the funding and commissioning of services for survivors has become increasingly complex (and devolved) over the past decade. Funding for VSS services comes from charitable trusts and local and national statutory sources, via health, local authorities and criminal justice organisations. This creates a dynamic network of responsibilities at the local level, which requires a high degree of collaboration between commissioners and providers. ${ }^{10}$ Complex commissioning arrangements also create challenges for providers in ensuring there are integrated, multidisciplinary care pathways for survivors.

One consequence of the recent drive to create a predominately free market model of commissioning is that the continued operation of some smaller organisations, who are adept at meeting the needs of specific groups at the local level, is increasingly under threat. ${ }^{16}$ In other areas, VSS providers are taking the initiative and collaborating between themselves, moving to common standards and seeking to join up services locally. Commissioners in turn are looking for an evidence to support these new approaches. ${ }^{1013} 17$ However, little is known about their effectiveness and how they affect the quality and nature of support for survivors.

\section{Evidence base for the value of VSS services for survivors}

There is little empirical evidence about the scope, range and effectiveness of VSS provision, or what survivors value and want from services. There is no systematic review evidence and systematic searches undertaken during the design and development of this study found no largescale, representative evidence. Studies use qualitative methods and typically draw on 'small' participant pools. The exceptions are one national survey with 395 adult survivors of child sexual abuse ${ }^{18}$ and evidence from 172 survivors of child sexual abuse submitted to the Independent Inquiry into Child Sexual Abuse. ${ }^{19}$ Survivors typically want timely, locally available services, a choice of therapy and long-term support from agencies taking a joined-up approach. ${ }^{19}$ They want to feel listened to, believed and respected, ${ }^{18}$ and the independence of VSS services from statutory services is seen as a key benefit. ${ }^{20}$ Counselling and psychotherapy are often cited as the most helpful services but waiting lists are often long and commissioned therapy may be time-limited. ${ }^{18}$ Many survivors need and use VSS services over many years. ${ }^{18}$

\section{STUDY AIMS AND OBJECTIVES}

This protocol reports a mixed-methods study involving survivors as co-researchers. The primary aims of the study are to:

1. Develop a comprehensive national profile of VSS services for survivors in England, giving voice to survivors' experiences and views.

2. Perform a comparative analysis of the range, scope and funding of services, survivors' service experiences, service models and approaches, service linkages and commissioning arrangements, in order to make policy and practice recommendations to strengthen the service response to survivors.

\section{METHODS AND ANALYSIS \\ Study design}

The study uses an explanatory sequential naturalistic mixed-methods design ${ }^{21}$ with two stages. In stage 1 , two national surveys of providers' and commissioners' views on designing and delivering VSS services will allow detailed mapping of service provision and commissioning in order to create a taxonomy of VSS services. Variations in the national picture will then be explored in stage 2 through four in-depth, qualitative case studies that will use the critical incident technique ${ }^{22}$ to explain the observed variations and understand the key contextual factors which influence service provision. Data in stage 2 will be collected via interviews with survivors and VSS staff and through documentary analysis. Figure 1 provides an overview of the study stages. 


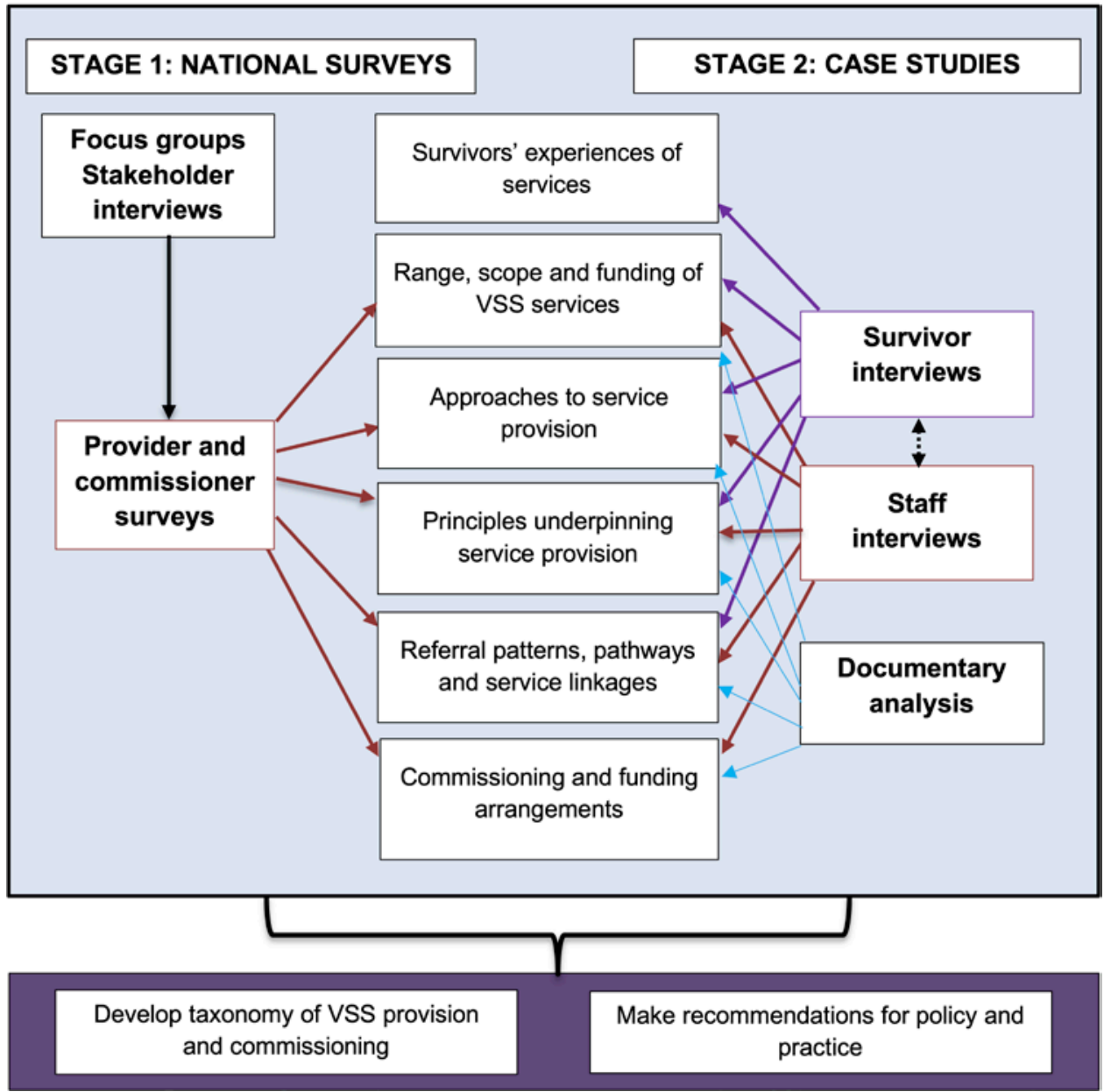

Figure 1 Study overview. VSS, voluntary sector specialist.

\section{Patient and public involvement}

Survivors' views informed this study from its inception and survivors are members of the study research team and study advisory group. Survivors will play a key role in the study's delivery and dissemination, providing critical, experiential knowledge about (some) survivors' needs and practical advice about engaging with survivors' networks and organisations. A VSS service will provide sensitisation training to all members of the research team at the onset of the study.

Four survivor co-researchers will be recruited to the study team. Co-research is a participatory and empowering methodology which will: amplify the voices of survivors; enhance the collection of meaningful data; empower survivors participating in the research; and promote learning and development of new skills. ${ }^{2324}$ Co-researchers will contribute to devising publicity and sensitive approaches to survivor recruitment and materials. Co-researchers will also advise on anxieties survivors may have in taking part in the study and how to address them. During stage 2, the co-researchers will carry out interviews with survivor participants alongside a member of the academic research team. The co-researchers will be trained and supported to develop skills in qualitative research methods and ethical issues. They will also be supported, through a process of supervision and peer debriefing, to identify and address their needs when working with survivors (as research participants).

\section{Theoretical orientation}

The study is informed by Billis and Glennerster's theory that the voluntary sector has unique features that give it a comparative advantage in the provision of human services compared with the statutory sector. ${ }^{25}$ These features include: flatter organisational structures with less distance and distinction between senior or decision-making staff and those on the front line; closeness to communities and being mission-led and driven by core values and purpose. By using this theory to underpin data collection and interpretation, the study will purposefully explore the nature of these distinctive features of voluntary organisations and their effect on the support provided to survivors.

\section{STAGE 1: NATIONAL PROVIDER AND COMMISSIONER SURVEYS}

In-depth surveys can explore aspects of a situation, seek explanations and provide data to test hypotheses..$^{26}$ These are important attributes for this study, which aims to map and explore national provision and commissioning arrangements for VSS services. Stage 1 involves the 
development (stage 1a) and dissemination (stage 1b) of national surveys for VSS providers and commissioners.

\section{Stage 1A: survey development}

Development of the provider and commissioner surveys will draw on the literature along with findings from 10 to 12 stakeholder telephone interviews and five focus groups, three for survivors and two for staff.

\section{Participants and recruitment}

Sexual violence survivors: Three survivor focus groups will be carried out, each comprising 6-10 participants drawn from diverse backgrounds in terms of age, gender, ethnicity and sexual orientation. Two focus groups will include adult participants (one for females only and one for males only). To ensure that young people's views can shape survey development, the third focus group will include 16/17year olds. Focus groups will be facilitated and coordinated by two VSS survivor organisations, who will identify potentially eligible survivors and distribute recruitment packs. Packs will include a participant information sheet (PIS), consent form and contact details for the research team. Recipients will have at least two weeks to consider participation before being contacted by the research team.

Senior providers and commissioners: 10-12 telephone interviews will be undertaken with national policy and commissioning leaders, along with two focus groups (8-12 participants per group) with senior staff involved in the provision and commissioning of sexual violence services: one in the north of England and one in the south/midlands. Although telephone interviews lack facial and body language cues, they can still produce rich data and can offer participants a more convenient way of taking part in research: this was an important consideration given the participant population of senior providers/commissioners. Participants must be currently in post or have been in post within the previous 12 months and will be invited through the study's advisory group, co-applicant team and recruited using social media strategies. Participants will be drawn from: VSS services, statutory services (eg, mental health, sexual health, drug and alcohol services) and commissioners of sexual violence VSS services from clinical commissioning groups, NHS England, local authorities and police and crime commissioners.

\section{Methods and analysis}

Focus groups: Focus groups produce rich, complex data about points of comparison, contrast and consensus between members of a group. ${ }^{27}{ }^{28}$ For the three survivor focus groups, a semi-structured topic guide will facilitate discussions. It will be used to explore survivors' views of: (1) their use of VSS services, (2) what they valued about these services, (3) why they might have chosen not to use some services, (4) how services could be improved and (5) ideas about topics to include in the surveys. The topic guide for the senior staff focus groups and telephone interviews will explore participants' views of: (1) the scope and nature of current VSS provision for survivors, (2) referral patterns, (3) service linkages, (4) commissioning arrangements and (5) topics to include in the surveys. The focus group and telephone interview data will be analysed and synthesised using a thematic analysis approach, as characterised by Braun and Clarke. ${ }^{29}$

\section{Stage 1B: provider and commissioner surveys} Participants and recruitment

There will be no sampling, with surveys sent electronically to the following groups:

1. Providers $(\mathrm{n}=500)$ : VSS service providers; Sexual Assault Referral Centre (SARC) managers; mental health NHS trusts; sexual health services, and NHS alcohol and drug services.

2. Commissioners $(n=400)$ : clinical commissioning groups (CCGs), local authority directors of public health; regional specialist health commissioners and police and crime commissioners.

Surveys will be distributed through our co-applicant and advisory groups' networks. Additionally, we will purposefully target national VSS and statutory organisations, using snowballing techniques to increase response rates. Recipients will be asked to forward the survey URL link to organisations in their local area by email or via professional accounts on social media platforms such as Twitter, Facebook and Instagram. To maximise response rates (target $30 \%+$ ), reminder emails will be sent 2-3 weeks after the initial survey request; telephone calls will target under-represented organisations or geographical areas and additional publicity will be sent via professional networks.

\section{Methods and analysis}

Surveys will be piloted in the West Midlands with 2-3 staff from VSS services and two-three commissioners from health, local authority or criminal justice. Both the provider and commissioner surveys will pursue the same lines of enquiry but with slightly different emphasis and wording of questions, in order to be of maximum relevance to their respective audiences (see box 1). Surveys will be concise and easy to fill in, with a mix of tick-box and free-text responses, taking about 20 minutes to complete. Because computer access may be limited in some of the smaller VSS organisations, paper surveys will be provided on request.

Surveys will be analysed descriptively to map patterns of VSS service provision across England. The analysis will identify key features of VSS services which are most variable across the country, to inform the development of the taxonomy of service provision and commissioning, which in turn will aid case study site selection. The views of respondents about the adequacy of services, possible improvements, and how commissioning is working will also be analysed descriptively, looking for differences and commonalities by stakeholder group (eg, comparing commissioners with providers or VSS providers with 
Box 1 Lines of enquiry for the provider and commissioner surveys

- How sexual violence is defined, principles underpinning service provision (eg, feminism or trauma-informed care), therapy models used and priority groups for service provision.

- Perceptions of what survivors want and need from VSS services, the benefits they derive and views about the value survivors put on VSS provision compared with statutory provision.

- What VSS services are provided, how they are delivered, by whom (for example, peer support, volunteers, professional staff) and to what quality standards.

- Criteria for accessing services, limits put on service use (for example, time limits/waiting lists), how outcomes are measured and examples of innovative practice.

- Referral patterns and pathways, how VSS services fit with each other and statutory services.

- How services respond to diverse population needs, unmet needs or service overlaps.

- Sources of funding and trends in funding over the past 5 years, including how funding pressures have been addressed locally and impacts on VSS provision.

- Factors influencing the pattern of local services for survivors in last 5 years.

statutory providers). The analysis will also identify key issues which merit further exploration through the case studies.

\section{STAGE 2: CASE STUDIES}

Stage 2 will build on the survey findings and use in-depth case study methods ${ }^{30}$ to explore how VSS services are provided and commissioned and the value and benefits survivors derive from these services. The case study work will include detailed analysis of VSS services in four geographical areas using a range of qualitative methods including: documentary analysis and interviews with VSS staff, staff working in the statutory sector, commissioners and survivors.

\section{Study sites and participants}

Site selection

The four study sites will be defined geographically by city or county boundaries, or groups of neighbouring districts. All VSS services for survivors located within that geographical boundary will be included, along with relevant statutory services. Site selection will be based on a realist approach to sampling, ${ }^{31}$ designed to generate information-rich cases, which can be compared strategically on variables which combine theory with real-world experience. A two-tier purposive sampling framework will, therefore, be used, using key variables identified from the national surveys. Tier 1 will relate to service provision and commissioning and a maximum diversity sample of sites will be selected using the taxonomies developed in stage 1 . Tier 2 will relate to location, with sites selected for maximum geographical spread across England and on the basis of population density (urban/ rural), demography (disadvantaged/affluent/mixed) and diversity (high/low black and minority ethnic populations). Once selected, each site will have a site reference group, led by a member of the research team. Representatives will be invited to take part from VSS organisations, SARCs, statutory services and commissioners from criminal justice, local authorities and health.

\section{Participants and recruitment: staff interviews}

There will be interviews with 17-19 staff per site, (68-76 in total across the four sites), including staff in senior and front-line roles, who may or may not have completed a stage 1 survey. Participants will also include frontline workers and/or managers/team leaders from: VSS services; SARCs; mental health services; alcohol and drug services; sexual health services, CCGs and specialised service commissioners; local authority commissioners and police and crime commissioners.

Potential participants will be identified in consultation with each site reference group. They will be sent an information pack, which will include: a PIS, consent form and contact details for the study team. Potential participants will be given at least 2 weeks to review the information before the study team make contact to ask if they would like to participate in an interview. Interviews will take place at participants' places of work or by telephone. The interviewers will be mindful that staff participants may also be survivors of sexual violence and will have information about local support services, should any participant require this.

\section{Participants and recruitment: survivor interviews}

Between 6-12 interviews will be undertaken with survivors across each site. Most interviews will be with survivors who have used VSS services in the past three years, so that experiences and views relate to recent/current service provision and are of maximum relevance (see table 1 for inclusion/exclusion criteria). Potential survivor participants will be sent an information pack, including: a PIS, consent form and contact details for the study team. It may be appropriate for local organisations to approach survivors who they think may be interested in taking part. This approach to recruitment will be supplemented with publicity going out through national organisations and local networks of professional and survivor-led fora. A variety of media will be used including leaflets, newsletters, emails and social media. Twitter and Facebook will be used as they have been found to be particularly effective for recruiting hard-to-reach groups ${ }^{32}$ Survivors interested in taking part will be able to contact the research team directly, on a secure dedicated mobile number, by post, email or on a confidential section of the study's website. They will be asked how and when they want to be contacted, in order that they cannot be identified incidentally. Some survivors may prefer to contact the team via a friend or member of staff they already know.

It is anticipated that most participants will elect to carry out the interviews at home because this is likely to be a 


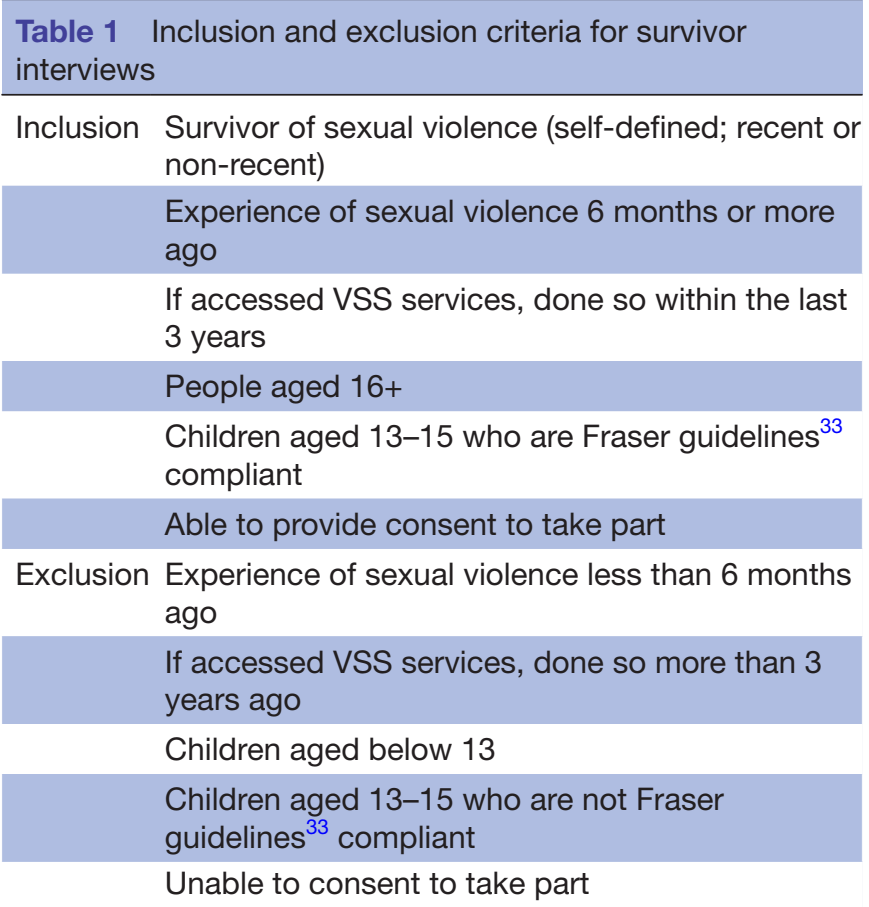

VSS, voluntary sector specialist.

more comfortable and convenient location than community venues; however, decisions about location will be made on case-by-case basis. Survivors can choose to have someone with them in the interview for support. Interviews will last between 30 and $60 \mathrm{~min}$ and will be audio recorded. A choice of male/female interviewer and co-researcher will be offered. As most survivors' experiences of sexual violence will be by male perpetrators, there will be more female than male interviewers available. An interpreter will be provided for survivors whose first spoken language is not English. Participants aged 16-17 years old will be invited to have a parent/carer or other appropriate adult (eg, support or social worker) with them when meeting with a member of the research team and taking part in an interview. For young people aged 13-15 years, the decision about participation will be made jointly between the young person, a parent/carer and the VSS staff member who knows them best. Young people aged 13-15 years must be in current receipt of VSS services at the time of interview. Fraser guidelines ${ }^{33}$ will be used to support the process of assessing young people's capacity to consent.

\section{Methods and analysis}

Documentary analysis

Documentary analysis will proceed alongside qualitative data collection so that points identified from documents can be explored in staff interviews. VSS and statutory service documents, such as commissioning plans, tender and service specifications, provider service publicity, referral pathways, staff profiles and outcome/performance data, will be analysed.

\section{Box 2 Semi-structured topic guide for survivor interviews}

What survivors felt they needed following the incident of sexual violence.

- Which VSS organisations survivors have made use of.

- Why specific services were usedand for how long.

- Which services were not used and why; and why one service was chosen over another.

- What differences there are in the ways VSS services have been provided.

- What values, benefits and limits are associated with VSS services, for themselves and their families/friends.

- What are the most and least helpful ways in which services were provided.

What gaps or overlaps there are in local services for survivors.

How services could be improved.

\section{Provider and commissioner interviews}

Staff interviews will be semi-structured and will be either face to face or over the telephone. An initial topic guide will be developed based on stage 1 results and will be piloted with two commissioners and two providers to ensure question validity.

\section{Survivor interviews}

Survivor interviews will be informal and will use the critical incident technique. ${ }^{22}$ This asks participants to recount experiences rather than talk in general or abstract terms. ${ }^{34}$ A narrative approach will encourage people to tell their stories about their service use, ${ }^{35}$ using a small number of open-ended stimulus questions. The narrative section of the interview will be followed by a second, semi-structured stage ${ }^{36}$ incorporating questions relating to researcher-defined lines of enquiry which have not already been covered by the participants (see box 2). Survivors will also be asked about why they have not taken up other VSS services on offer locally, why they chose one service over another, and how the ways in which services are provided might make services more or less acceptable to other survivors. Although the focus of the interviews is on survivors' views and experiences of specialist services, survivors (who may also be staff) may discuss topics that they experience as emotive or distressing. The researchers will follow the principles of the McCosker et al distress protocol to guide how they identify and respond to participants' distress or discomfort. ${ }^{37}$

\section{Data analysis}

Documents will be analysed using conventional content analysis. ${ }^{38}$ Staff and survivor interviews will be recorded digitally and professionally transcribed, with transcripts checked against audio files for accuracy. Coding and analysis will use NVivo software. Survivor interview data will be analysed using an inductive classification system designed for critical incident data. ${ }^{22}$ Although the analysis will be primarily data led and inductive, ${ }^{39}$ additional analysis will compare survivors' views about the value of services against the principles of trauma-informed care. ${ }^{40}$ The 
same analytical processes will be undertaken for a site-bysite analysis of the staff interviews, using thematic analysis $^{29}$ and sub-group analysis by professional role where appropriate. Data from the staff and survivor interviews, and the documentary analysis will then be combined, building a detailed picture of each site. We will draw on Billis and Glennerster's theory of the distinctiveness of human service voluntary sector provision to inform our analysis.

A preliminary cross-site analysis will use a matrix to compare themes across sites and groups (survivors, staff and commissioners). This will triangulate themes from multiple sources; assess the frequency with which themes occur across sites; explore patterns and plausible explanations; and identify themes with the greatest explanatory power. The context, facilitators and barriers to VSS service delivery and commissioning will also be explored.

\section{DATA INTEGRATION}

Qualitative and quantitative data will be integrated from all stages in order to establish key themes and concepts; identify different configurations and patterns of service provision; and, explore survivors' experiences of these services. Triangulation methodology originally developed for analysing mixed-methods results from clinical trials will be used. ${ }^{41}$ This will involve:

1. Methodological triangulation, comparing data using different methods, for example, comparing survivors' experiences using data from focus groups and interviews.

2. Data triangulation, comparing quantitative with qualitative data, for example, comparing quantitative results about service provision and commissioning from the quantitative surveys with the qualitative case study interviews.

3. Investigator triangulation, comparing the analysis of the same data by different team members, including different interpretations from individual academics and co-researchers.

Key findings in the form of short written statements from the separate analysis of each dataset will be discussed; integrated into a matrix; triangulated by members of the research team individually; then, using different categories of agreement, collectively shared and discussed by the research team.

\section{DISSEMINATION}

\section{Ethical issues and governance}

In line with the sequential and co-produced study design, further ethical approvals will be sought from the Health Research Authority and relevant local authority and police and crime commissioner ethical review boards as the study progresses.

Principal ethical issues include:

- Identifying and responding to the practical, emotional and social needs of participants (particularly survivors), so that research engagement is a meaningful, informed and positive experience.

- Identifying and responding to the individual needs of the team's survivor co-researchers and co-applicants, including reducing the risk of re-traumatisation or 'over-involvement'.

- Ensuring the confidentiality and anonymity of participants' data and its safe storage, in line with best practice principles of data protection and archiving.

\section{Dissemination and expected outputs}

Dissemination activities will include an interim written report and workshop reporting the national survey results. End-of-study activities include case study site workshops; regional workshops; good practice guides; a policy briefing; project report; bitesize findings; webinars; academic articles and conference presentations.

\section{DISCUSSION}

This study will generate evidence about what survivors want from and value about services and how VSS services should be commissioned and provided to support survivors to thrive in the long term. Commissioners and providers will be able to compare their local services against the national picture in order to configure services optimally. In the longer term, improvements in survivors' well-being should lead to reductions in service demands.

\section{Author affiliations \\ ${ }^{1}$ Institute of Applied Health Research, University of Birmingham, Birmingham, UK \\ ${ }^{2}$ Social Science ans Systems in Health, University of Warwick, Coventry, UK \\ ${ }^{3}$ Avon and Somerset Constabulary, Portishead, UK \\ ${ }^{4}$ The Survivors Trust, Rugby, UK \\ ${ }^{5}$ School of Social Work and Social Care, University of Birmingham, Birmingham, UK \\ ${ }^{6}$ School of Nursing, College of Medical and Dental Sciences, University of Birmingham, Birmingham, UK \\ ${ }^{7}$ Birmingham Women's and Children's Hospital NHS Foundation Trust, Birmingham, UK}

Contributors GC led and co-ordinated the design and development of the study and protocol. All members of the coapplicant team contributed to, and reviewed, the study design and protocol (CB-J, SD, JH, AJ, FM, PP, JS, DS and JT). The study research fellows, $\mathrm{LI}$ and $\mathrm{CG}$, contributed to drafting and refinement of the study protocol and development of this paper.

Funding The protocol reports a study funded by the National Institute for Health Research (NIHR), Health Services and Delivery Research (HS\&DR) funding stream (Ref: 18/02/27).

Disclaimer The views expressed are those of the author(s) and not necessarily those of the NIHR or the Department of Health and Social Care.

Competing interests None declared.

Patient consent for publication Not required.

Ethics approval Ethical approval (application ERN-19-1152) has been obtained from the University of Birmingham research ethics committee for stage 1a) survey development.

Provenance and peer review Not commissioned; peer reviewed for ethical and funding approval prior to submission.

Open access This is an open access article distributed in accordance with the Creative Commons Attribution Non Commercial (CC BY-NC 4.0) license, which permits others to distribute, remix, adapt, build upon this work non-commercially, and license their derivative works on different terms, provided the original work is 
properly cited, appropriate credit is given, any changes made indicated, and the use is non-commercial. See: http://creativecommons.org/licenses/by-nc/4.0/.

\section{ORCID iD}

Gill Combes http://orcid.org/0000-0001-6829-4621

\section{REFERENCES}

1 World Health Organization. Global and regional estimates of violence against women: prevalence and health effects of intimate partner violence and non-partner sexual violence. World Health Organization, 2013.

2 Logan TK, Walker R, Cole J. Silenced suffering: the need for a better understanding of partner sexual violence. Trauma Violence Abuse 2015;16:111-35.

3 ONS. Crime in England and Wales: year ending March 2018. London: Office for National Statistics, 2018.

4 Home Office. A report by Baroness Vivien stern CBE of an independent review into how rape complaints are handled by public authorities in England and Wales. London: Home Office, 2010.

5 Mason F, Lodrick Z. Psychological consequences of sexual assault. Best Pract Res Clin Obstet Gynaecol 2013;27:27-37.

6 Hester M, Lilley S-J. More than support to court: rape victims and specialist sexual violence services. Int Rev Vict 2018;24:313-28.

7 Brooker C, Tocque K, Paul S. Assessment of the mental health status of a one year cohort attending a two sexual assault referral centres in England. J Forensic Leg Med 2018;54:44-9.

8 Felitti VJ, Anda RF, Nordenberg D, et al. Relationship of childhood abuse and household dysfunction to many of the leading causes of death in adults: the adverse childhood experiences (ACE) study. Am J Prev Med 2019;56:774-86.

9 Loya RM. Rape as an economic crime: the impact of sexual violence on survivors' employment and economic well-being. J Interpers Violence 2015;30:2793-813.

10 All Party parliamentary group, sexual violence report into the funding and commissioning of sexual violence and abuse services 2018.

11 HM Government,. Cross government action plan on sexual violence and abuse. London: HM Government, 2006.

12 HM Government,. Ending violence against women and girls. London: HM Government, London, 2016.

13 NHS England. Securing excellence in commissioning sexual assault services for people who experience sexual violence. England: Leeds; NHS, 2013.

14 NHS England. Strategic direction for sexual assault and abuse services. Lifelong care for victims and survivors 2018-2023. London: NHS England, 2018.

15 Walker IF, Leigh-Hunt N, Lee ACK. Redesign and commissioning of sexual health services in England - a qualitative study. Public Health 2016;139:134-40.

16 Simmonds L. The impact of local commissioning on victim services in England and Wales: an empirical study. Int Rev Vict 2019:25:181-99.

17 NHS England. Public health functions to be exercised by the NHS England. Service specification No.30, sexual assault referral centres. England: Leeds; NHS, 2016.

18 Smith N, Dogaru C, Ellis F. Hear me. Believe me. Respect me. A survey of adult survivors of child sexual abuse and their experiences of support services. University Campus Suffolk, 2015.

19 Independent inquiry into child sexual abuse, consultation on the impacts of child sexual abuse and experiences of support services. A summary of responses received. IICSA 2016.
20 Robinson A, Hudson K. Different yet complementary: two approaches to supporting victims of sexual violence in the UK. Criminology \& Criminal Justice 2011;11:515-33.

21 Creswell JW, Clark VL. Designing and conducting mixed methods research. Sage, 2017.

22 Cormack D. The research process in nursing. Oxford: Blackwell Science, 2000

23 Bradbury-Jones C, Taylor J. Engaging with children as coresearchers: challenges, counter-challenges and solutions. Int J Soc Res Methodol 2015;18:161-73.

24 Bradbury-Jones C, Isham L, Taylor J. The complexities and contradictions in participatory research with vulnerable children and young people: a qualitative systematic review. Soc Sci Med 2018;215:80-91.

25 Billis D, Glennerster $\mathrm{H}$. Human services and the voluntary sector: towards a theory of comparative advantage. J Soc Policy 1998;27:79-98.

26 Kelley K, Clark B, Brown V, et al. Good practice in the conduct and reporting of survey research. Int J Qual Health Care 2003;15:261-6.

27 Robinson N. The use of focus group methodology - with selected examples from sexual health research. $J$ Adv Nurs 1999;29:905-13.

28 Kitzinger J. Focus group research: using group dynamics. Qual Res Health 2005;56.

29 Braun V, Clarke V. Using thematic analysis in psychology. Qual Res Psychol 2006;3:77-101.

30 Yin RK. Case study research and applications. Design and methods. Los Angeles: Sage, 2018.

31 Emmel N. Sampling and choosing cases in qualitative research: a realist approach. Sage, 2013.

32 Thornton L, Batterham PJ, Fassnacht DB, et al. Recruiting for health, medical or psychosocial research using Facebook: systematic review. Internet Interventions 2016;4:72-81.

33 NSPCC. Gillick competency and Fraser guidelines. Balancing children's rights with the responsibility to keep them safe from harm. London: NSPCC, 2018.

34 Britten L. The enhanced critical incident technique: using semistructured interviews to work with vulnerable and marginalized populations. Sage, 2014

35 Anderson C, Kirkpatrick S. Narrative interviewing. Int J Clin Pharm 2016;38:631-4.

36 Scheibelhofer E. Combining Narration-Based interviews with topical interviews: methodological reflections on research practices. Int $J$ Soc Res Methodol 2008;11:403-16.

37 McCosker H, Barnard A, Gerber R. Undertaking sensitive research: issues and strategies for meeting the safety needs of all participants. Forum Qualitative Sozialforschung/Forum: Qualitative Social Research 2001;2, No. 1.

38 Hsieh H-F, Shannon SE. Three approaches to qualitative content analysis. Qual Health Res 2005;15:1277-88.

39 Stake RE. Qualitative Case Studies. In: Denzin NK, Lincoln YS, eds. The SAGE Handbook of qualitative research. California, USA: SAGE, 2005: 443-66.

40 Elliott DE, Bjelajac P, Fallot RD, et al. Trauma-informed or traumadenied: principles and implementation of trauma-informed services for women. J Community Psychol 2005;33:461-77.

41 Tonkin-Crine S, Anthierens S, Hood K, et al. Discrepancies between qualitative and quantitative evaluation of randomised controlled trial results: achieving clarity through mixed methods triangulation. Implement Sci 2015;11. 